\title{
Optimization of Ridge Waveguide Structure for Temperature Sensor Application Using Finite Difference Method
}

\author{
Ian Yulianti ${ }^{1,{ }^{*}}$, Ngurah Made D. $\mathrm{P}^{1}$, Yuni Lestiyanti ${ }^{1}$, Ojo Kurdi ${ }^{2}$ \\ ${ }^{1}$ Physics Department, Faculty of Mathematics and Natural Sciences, Universitas Negeri Semarang, \\ Central Java, Indonesia \\ ${ }^{2}$ Department of Mechanical Engineering, Faculty of Engineering, Universitas Diponegoro, Central \\ Java, Indonesia
}

\begin{abstract}
This work presents optimization of the dimension of ridge waveguide structure to be used as temperature sensor. The objective of the work is to obtain an optimum dimension of ridge waveguide so that it provides high sensitivity to temperature. Ridge waveguide structure was chosen since it has strong light confinement and low bending loss. The optimization was done by simulating the temperature distribution and electric field distribution inside the waveguide using Finite Difference Method (FDM). The simulations were done for various waveguide width and waveguide thickness. The results showed that as the width increased, the sensitivity decreased. The optimum ranges of waveguide dimensions are between $10 \mu \mathrm{m}$ to $20 \mu \mathrm{m}$ and $15 \mu \mathrm{m}$ to $25 \mu \mathrm{m}$ for width and thickness, respectively.
\end{abstract}

\section{Introduction}

Temperature sensors have attracted much attention due to its important role in various applications such as environmental monitoring, chemical industry and automotive industry. Conventional methods have been used to develop temperature sensor such as mechanical and electrical method. However, conventional methods are not suitable for conductive and corrosive environment [1]. Therefore, optical temperature sensors have been proposed to replace the conventional sensors. The most common optical temperature sensor is optical fiber-based sensor with various methods such as interference method [1], wavelength modulation method [2] and intensity modulation method [3]. Optical fiber sensors have advantages of free from electromagnetic interference, suitable for hazardous environment and can be arranged in multiplexed array. However, optical fiber sensor suffers from bending loss that degrades the accuracy, especially for intensity modulation based sensor.

\footnotetext{
* Corresponding author: ianyulianti@gmail.com
} 
Besides optical fiber-based sensor, optical temperature sensor can be realized by using optical waveguide. Compared to optical fiber, optical waveguide has some advantages such as robust, has high sensitivity, easy light intensity measurement and can be realized in onchip sensor[4]. Various optical waveguide sensors have been proposed for various applications such as for refractive index measurement [5-7], and biosensor [8, 9]. The published waveguide-based optical sensor were proposed by implementing various method such as surface plasmon resonance [4, 10, 11], Bragg grating [7] and mode coupling [6]. For temperature measurement, waveguide-based optical sensor has been proposed using intensity modulation method by applying curved waveguide [12]. It was shown that by decreasing the curvature radius, the sensitivity increased. However, decreasing curvature radius limits the sensor's working range. Meanwhile, the most recent research proposed a temperature sensor by using prism-waveguide with silicon acrylate as sensing layer [13]. The working principle is based on detection of lateral shift of the reflected light. Despite its high accuracy, the measurement procedure is not straightforward. Simple measurement can be done by adopting intensity modulation method using simple waveguide structure such as ridge waveguide. Hence, in this work, we investigate the sensitivity of ridge waveguide and optimize its dimension for temperature sensor application

\section{Measurement Principles and Waveguide Structure}

Light propagation in waveguide highly depends on refractive index of waveguide material which are cladding and core material. High refractive index difference between core and cladding will result in high light confinement which is indicated by high propagation constant [14]. Due to thermo-optic nature of material, refractive index is thus changed as the waveguide's temperature change. Therefore, the propagation constant and, consequently, the output light intensity transmitted by the waveguide will also change as temperature changes. Hence, by measuring the light intensity, the waveguide's surrounding temperature can be determined.

Among various waveguide types, ridge waveguide structure is interesting for temperature sensor application since the core is directly exposed to environment i.e. the core is not surrounded by cladding material as shown in Figure 1. Moreover, ridge waveguide has strong light confinement and low bending loss. As material, polymer was chosen since it has high thermo-optic coefficient $\left(-1,7 \times 10^{-4} / \mathrm{K}\right)$ that allows high refractive index change due to small change of temperature.

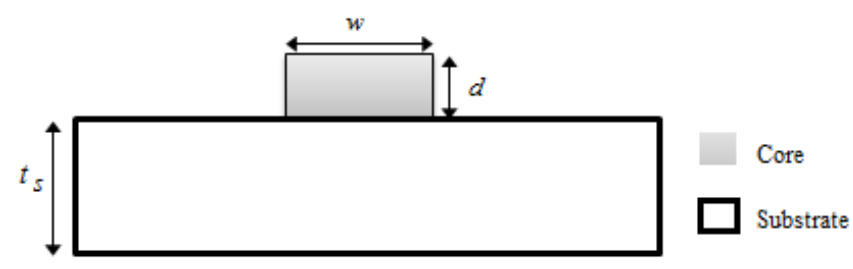

Fig. 1. Cross-sectional view of ridge waveguide

\section{Optimization Method}

Optimization of waveguide dimension was done by simulating the temperature effect to propagation constant of the waveguide. The first stage of the optimization was performing simulation of temperature distribution inside the waveguide by solving heat transfer equation using Finite Difference Method (FDM). After temperature distribution simulation, the next stage was calculating the waveguide refractive index change due to the 
temperature change by using thermo-optic coefficient formula. Then, the electric field distribution inside the waveguide and the propagation constant were obtained by conducting modal analysis. The simulations were done for various temperature values and various waveguide widths and thicknesses. The sensitivity of each waveguide dimension was obtained by plotting the propagation constant vs. temperature.

\subsection{Temperature Distribution Simulation}

Temperature distribution inside waveguide was obtained by solving heat transfer equation in constant thermal conductivity as defined by

$$
k \nabla^{2} T+Q(x, y, z, t)=\rho c_{p} \frac{\partial T}{\partial t}
$$

where $\rho$ is the material density, $c_{p}$ is the specific heat, $k$ is the thermal conductivity which is $0.17 \mathrm{~W} / \mathrm{mK}$ for polymer and $Q(x, y, z, t)$ is the heat generation rate per unit volume. Since the length of the waveguide is much higher than the width, then the heat transfer simulation was done in two dimensional analyses. The simulation was also done for steady state heat conduction, thus equation (1) can be simplified as

$$
k\left(\frac{\partial^{2} T(x, y)}{\partial x^{2}}+\frac{\partial^{2} T(x, y)}{\partial^{2} y^{2}}\right)+Q(x, y, z, t)=0
$$

Equation (2) was solved numerically using FDM by applying centered-difference approximation. In the FDM, the domain of analysis is discretized with a rectangular grid of points, which may be of constant or variable spacing. By using constant spacing, Equation (2) becomes

$$
4 T_{i, j}=T_{i+1, j}+T_{i-1, j}+T_{i, j+1}+T_{i, j-1}+\frac{\mathrm{Q} \Delta x \Delta y}{k}
$$

where $\Delta x$ and $\Delta y$ are the grid size. As boundary condition, all sides of the computation window were assumed to be subjected to convection while the bottom surface is considered as heat sink.

The temperature change was then used to calculate the refractive index change of waveguide by using thermo-optic coefficient (Toc) formula which is defined as

$$
T o c=\frac{d n}{d T}=-\left(\frac{\sigma \partial n}{\partial \sigma}\right)_{T} \eta+\left(\frac{\partial n}{\partial T}\right)_{\sigma}
$$

where $\eta$ is the coefficient of volume expansion of material, $n$ is the refractive index, and $\sigma$ is the material density [15].

\subsection{Modal Analysis}

In waveguide design process, modal analyses is an important stage since it determines the value of propagation constant $(\beta)$ and the corresponding modal pattern for each desired mode. The equation to be solved in modal analyses is the Maxwell equation which is defined by

$$
\frac{\partial^{2}}{\partial x^{2}} E(x, y)+\frac{\partial^{2}}{\partial y^{2}} E(x, y)+\left[k_{o}^{2} n(x, y)^{2}-\beta^{2}\right] E(x, y)=0
$$

for plane wave that propagates along $z$ direction with propagation constant $\beta . E(x, y)$ is the electric field, $k_{o}$ is a free space wave number and $n$ is the index of refraction. By double integrating Equation (5) and multiplying with $E(x, y)$, then $\beta$ can be written as 


$$
\beta^{2}=\frac{\iint E\left[\frac{\partial^{2}}{\partial x^{2}} E+\frac{\partial^{2}}{\partial y^{2}} E+k_{o}^{2} n^{2} E\right] d x d y}{\iint E^{2} d x d y}
$$

Like temperature distribution simulation, Equation (5) and (6) were also discretized by using FDM with centered-difference approximation to obtain the propagation constant and electric field distribution inside waveguide.

\section{Results and Discussions}

The simulations were done for various temperature values which are in the range of $30^{\circ} \mathrm{C}$ $100^{\circ} \mathrm{C}$ with increment of $10^{\circ} \mathrm{C}$. All stages were then repeated for various waveguide dimensions. In this work, the width of the waveguide $(w)$ was incremented from $10 \mu \mathrm{m}$ to $50 \mu \mathrm{m}$ with increment of $5 \mu \mathrm{m}$. The thickness $(d)$ was also varied from $10 \mu \mathrm{m}$ to $50 \mu \mathrm{m}$ with increment of $5 \mu \mathrm{m}$. Meanwhile, the substrate was maintained at thickness of $30 \mu \mathrm{m}$. The refractive index of core $\left(n_{c}\right)$ and substrate $\left(n_{s}\right)$ are 2.65 and 1.489 , respectively.

The results showed that as temperature increased, the electric field inside waveguide was less confined in the waveguide as indicated by more scattered electric field distribution as shown in Figure 2. Therefore, it results in low propagation constant. The less confined waveguide was occurred due to the decrease of refractive index which is resulted from negative thermo-optic coefficient of polymer.

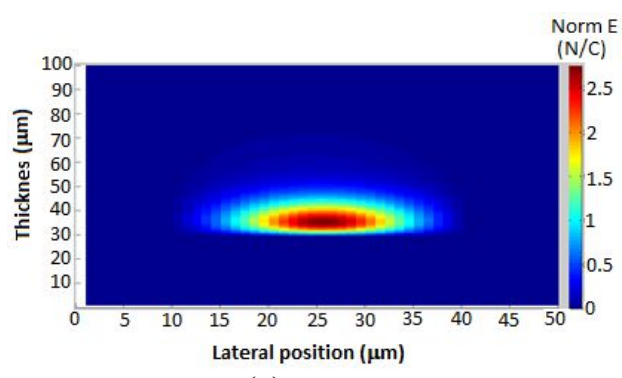

(a)

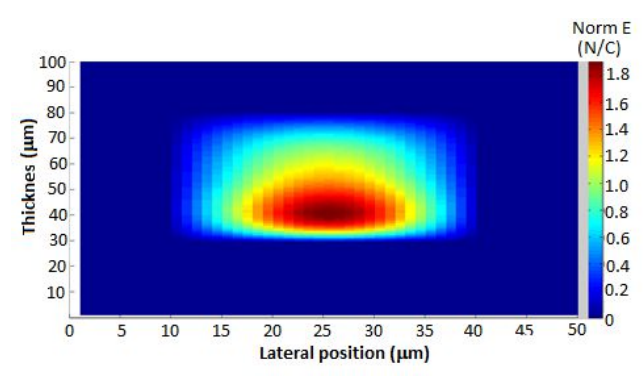

(b)

Fig. 2. Electric field distribution inside waveguide with $d=50 \mu \mathrm{m}$ and $w=30 \mu \mathrm{m}$ for environment temperature of $10^{\circ} \mathrm{c}(a)$ and $30^{\circ} \mathrm{C}(b)$.

As shown in Figure 3(a), for waveguide width of $10 \mu \mathrm{m}, 30 \mu \mathrm{m}$ and $50 \mu \mathrm{m}$ and thickness of $50 \mu \mathrm{m}$, the propagation constant decreased as temperature increased which results in negative curve slope. The trend is also valid for all waveguide dimensions. Therefore, the sensors have negative sensitivity. The sensitivity for various waveguide widths and fixed thickness is shown in Figure 3(b). It is shown that by increasing waveguide width up to $25 \mu \mathrm{m}$, the sensor sensitivity almost maintain at value of around $-680 \mathrm{rad} / \mathrm{m}^{\circ} \mathrm{C}$. However, for width of above $25 \mu \mathrm{m}$, the sensitivity degraded significantly. The highest sensitivity was observed at width of $20 \mu \mathrm{m}$, which is $-685.71 \mathrm{rad} / \mathrm{m}^{\circ} \mathrm{C}$. 


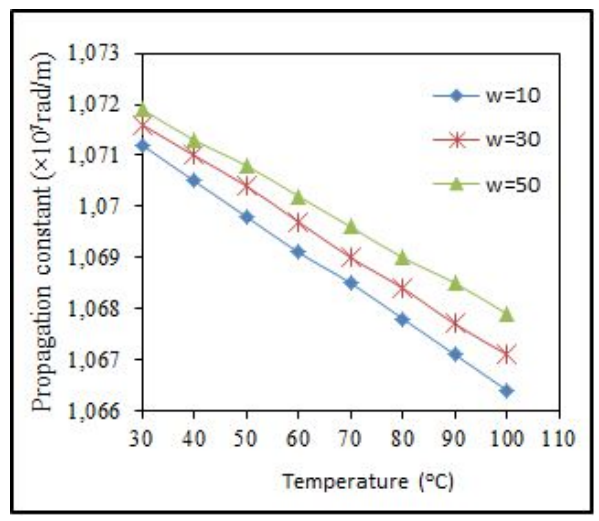

(a)

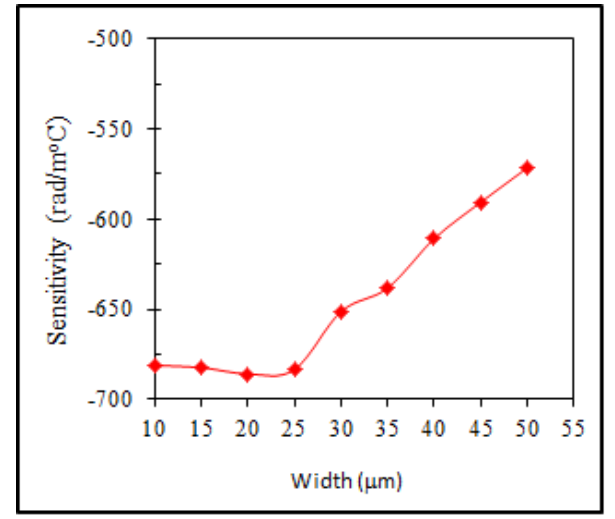

(b)

Fig. 3. Temperature effect to propagation constant for various widths $(d=50 \mu \mathrm{m})(a)$ and sensitivity vs. width for $d=50 \mu \mathrm{m}(b)$.

Sensitivity's for various widths and various thicknesses are depicted in Figure 4. It is shown that for all thickness values, waveguide widths of below $20 \mu \mathrm{m}$ provide high sensitivity as shown by the black contour on the graph. However, the highest sensitivity was achieved at waveguide width of $10 \mu \mathrm{m}$ and thickness of $15 \mu \mathrm{m}, 20 \mu \mathrm{m}$ and $25 \mu \mathrm{m}$ which is $-691.67 \mathrm{rad} / \mathrm{m}^{\circ} \mathrm{C}$. Therefore, the optimum ranges of the ridge waveguide dimensions are between $10 \mu \mathrm{m}$ to $20 \mu \mathrm{m}$ and $15 \mu \mathrm{m}$ to $25 \mu \mathrm{m}$ for width and thickness, respectively. Hence, it can be concluded that small waveguide dimensions provide high temperature sensitivity. The results are reasonable since the effect of environment temperature will be higher in small waveguide than that in large waveguide. As consequence, high change of propagation constant will occur in small waveguide.

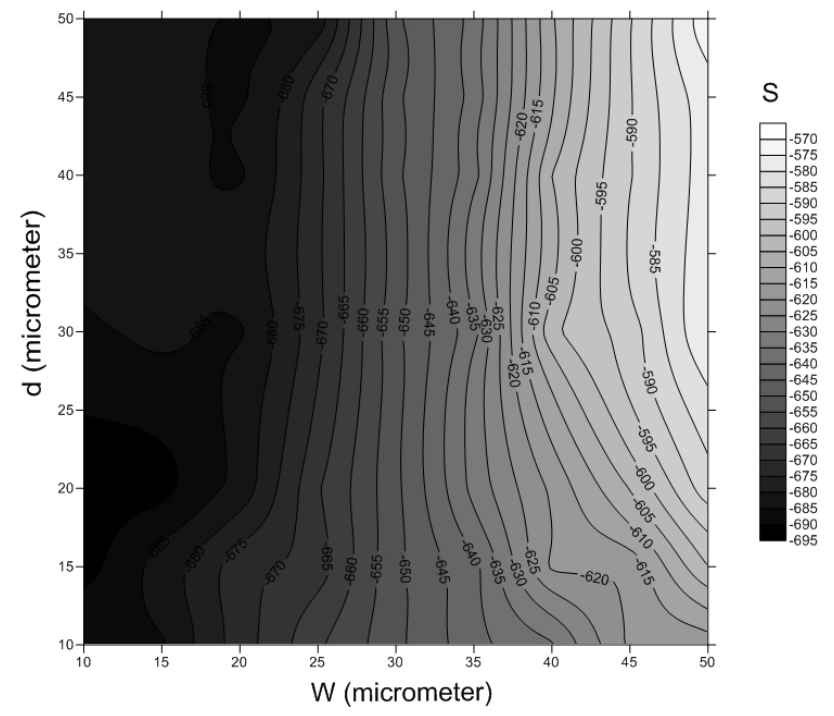

Fig. 4. Temperature sensitivity of ridge waveguide for various widths and various thicknesses. 


\section{Conclusions}

Simulation and optimization of ridge waveguide have been conducted. It was shown that ridge waveguide provide high sensitivity to temperature. The optimum dimension for temperature sensor application is in the range of $10 \mu \mathrm{m}$ to $20 \mu \mathrm{m}$ and $15 \mu \mathrm{m}$ to $25 \mu \mathrm{m}$ for width and thickness, respectively. Therefore, ridge waveguide is a good candidate to be developed as temperature sensor. However, response time of the sensor need to be further investigated which will be carried out in our future work.

\section{References}

[1] L. Ma, Z. Kang, Y. Qi, and S. Jian, Optik. 126 (2015).

[2] J. Li, L. Gai, H. Li, and H. Hu, Sensors and Actuators A: Physical. 263 (2017).

[3] A. T. Moraleda, C. V. García, J. Z. Zaballa, and J. Arrue, Sensors. 13 ( 2013).

[4] W. Du and F. Zhao, Materials Letters. 115 (2014).

[5] B. Hooda and V. Rastogi, Optik - International Journal for Light and Electron Optics. 143 (2017).

[6] G. C. Yadav, G. Sharma, S. Kumar, Deepak, R. Kumar, S. Prasad, et al., Optik International Journal for Light and Electron Optics. 138 (2017).

[7] N. Saha, A. Kumar, and A. Mukherjee, Optics Communications. 396 (2017).

[8] R. Horvath, H. C. Pedersen, N. Skivesen, and N. B. Larsen, "Optical waveguide sensor for monitoring living cell morphology," in CLEO/Europe. 2005 Conference on Lasers and Electro-Optics Europe, 2005., 2005, pp. 630-630.

[9] Y. Fan, Y. Ding, H. Ma, N. Teramae, S. Sun, and Y. He, Biosensors and Bioelectronics. 67 (2015).

[10] R. Levy and S. Ruschin, Sensors and Actuators B: Chemical. 124, 2, (2007).

[11] P. K. Teotia and R. S. Kaler, Optics Communications. 395 (2017).

[12] M. Remouche, R. Mokdad, A. Chakari, and P. Meyrueis, Optics \& Laser Technology 39 (2007).

[13] Y. Chen, HongRen, and TingtingTang, Optik. 124 (2013).

[14] I. Yulianti, A. S. M. Supa'at, S. M. Idrus, and A. B. Mohammad, "Optimization of buried type waveguide for single-mode operation," presented at the Proceedings of the Fifth IASTED International Conference on Communication Systems and Networks, Langkawi, Malaysia, 2008.

[15] M.B.J Diemeer, Optical Materials. 9 (1998). 